\title{
Overview of European legislation on informed consent for neonatal research
}

Euricon, a European Union funded study, ${ }^{1}$ had the objective of examining the issue of obtaining informed consent for neonatal research across Europe. As part of that project, legal representatives from these countries (see acknowledgements) were invited to a colloquium to report, discuss, and analyse European legal frameworks governing informed consent for neonatal research. The first author (PDV) presented a general overview of European law, which is the basis for this report. Furthermore, specific supplemental information from different countries was given by legal representatives from these countries, and is summarised in table 1 .

Legally and ethically, informed consent of the subject is a requirement for biomedical research. However, there are differing interpretations in European Law of this requirement for neonatal research. Increasingly, to answer many neonatal research questions, there is a need for multinational recruitment to randomised controlled trials and studies, and thus a need for investigators to be aware of the differing, relevant, national legislation.

The aim of this paper is to discuss areas of consistency and inconsistency in the law or practice governing informed consent for neonatal research in ten European countries (Denmark, Finland, France, Germany, Greece, Ireland, Norway, Spain, Sweden, and the United Kingdom). In particular, the following areas are examined: whether there is specific law governing informed consent for research and, if so, what it is; the ethical review system, whether there are specific requirements for consent, whether benefit for the individual child is a specific requirement, and whether research of no direct benefit (so called non-therapeutic research) is legally permitted in minors-for example, the taking of extra blood samples purely for research purposes.

The law governing neonatal research across Europe A variety of laws and a plethora of ethical and professional guidelines govern the conduct of research within individual countries and across Europe.

The Declaration of Helsinki, ${ }^{2}$ first adopted by the World Medical Assembly in 1964 and last amended in 1996, acts as a universal ethical guide. Throughout Europe, research ethics committees require that research trials and studies comply with this Declaration. Further guidance is proffered in the 1989 United Nations Convention on the Rights of the Child.

The principles of the Declaration of Helsinki have been incorporated into the International Conference on Harmonisation Guidelines for good clinical practice (ICH GCP). ${ }^{3}$ United States and Japan, as well as the European regulatory authorities, have adopted the latter, to which all regulatory trials must comply in order to be granted authorisation to market a medicinal product.

A further measure to introduce harmonisation across Europe for the conduct of research is the Council of Europe's 1997 Convention for the Protection of Human
Rights and Dignity of the Human Being with regard to the Application of Biology and Medicine. ${ }^{4}$

Article 16 of the Convention sets out general conditions to research on human beings:

(a) there is no alternative of comparable effectiveness to research on humans;

(b) the risks are not disproportionate to the potential benefits of the research;

(c) the research project has been approved by the competent body after independent examination of its scientific merit, including assessment of the importance of the aim of the research, and multidisciplinary review of its ethical acceptability;

(d) the persons undergoing research have been informed of their rights and the safeguards prescribed by law for their protection;

(e) the necessary consent has been given expressly, specifically and is documented. Such consent may be freely withdrawn at any time.

The Convention further sets out restrictions for research on persons not able to consent, such as neonates. This research may only be undertaken when:

(a) the above mentioned general conditions on research are fulfilled;

(b) the results of the research have the potential to produce real and direct benefit to their health;

(c) research of comparable effectiveness cannot be carried out on individuals capable of giving consent;

(d) the necessary authorisation has been given specifically and in writing.

Exceptionally, research that does not have the potential to produce results of direct benefit to the health of the child is authorised in Article 17 of the Convention if additional conditions are satisfied:

(a) the research has the aim of contributing, through significant improvement in the scientific understanding of the individual's condition, disease or disorder, to the ultimate attainment of results capable of conferring benefit to the person concerned or to other persons in the same age, category or afflicted with the same disease or disorder or having the same condition;

(b) the research entails only minimal risk and minimal burden for the individual concerned.

A new European Union Directive is being amended currently which includes both the ICH GCP guidelines and the above Convention. It is designed to regulate clinical trials further, but probably will be limited to those used to develop medicinal products within the pharmaceutical industry and thus will harmonise regulation of this type of trial across Europe.

Non-pharmaceutical research is subject to less standardisation. For these, investigators should comply with the Declaration of Helsinki if not also the above Convention for the Protection of Human Rights. However, different cultural and religious contexts, professional practices and historical antecedents have resulted in variations in practice and law throughout Europe. ${ }^{5}$ Differences and 


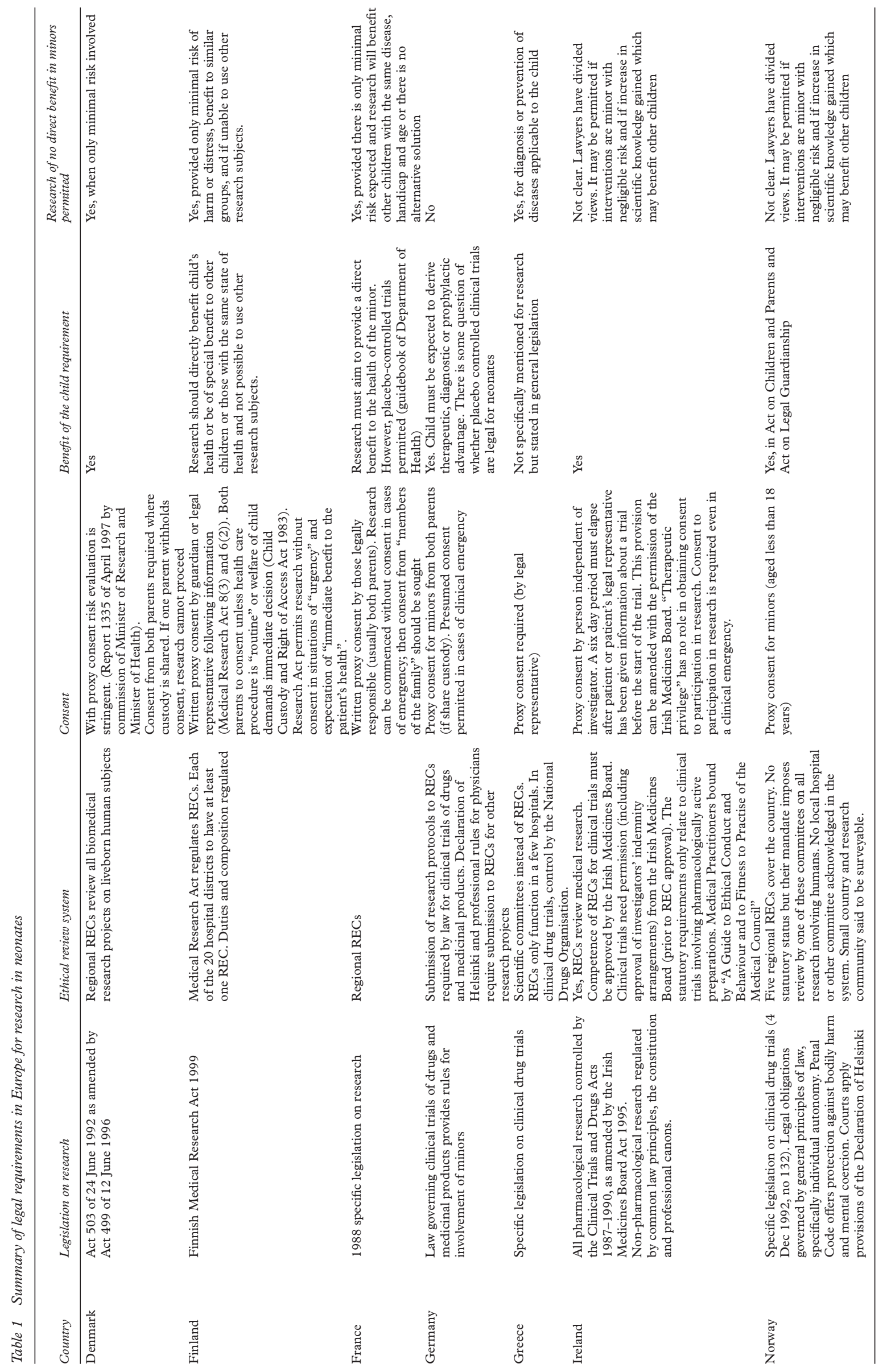




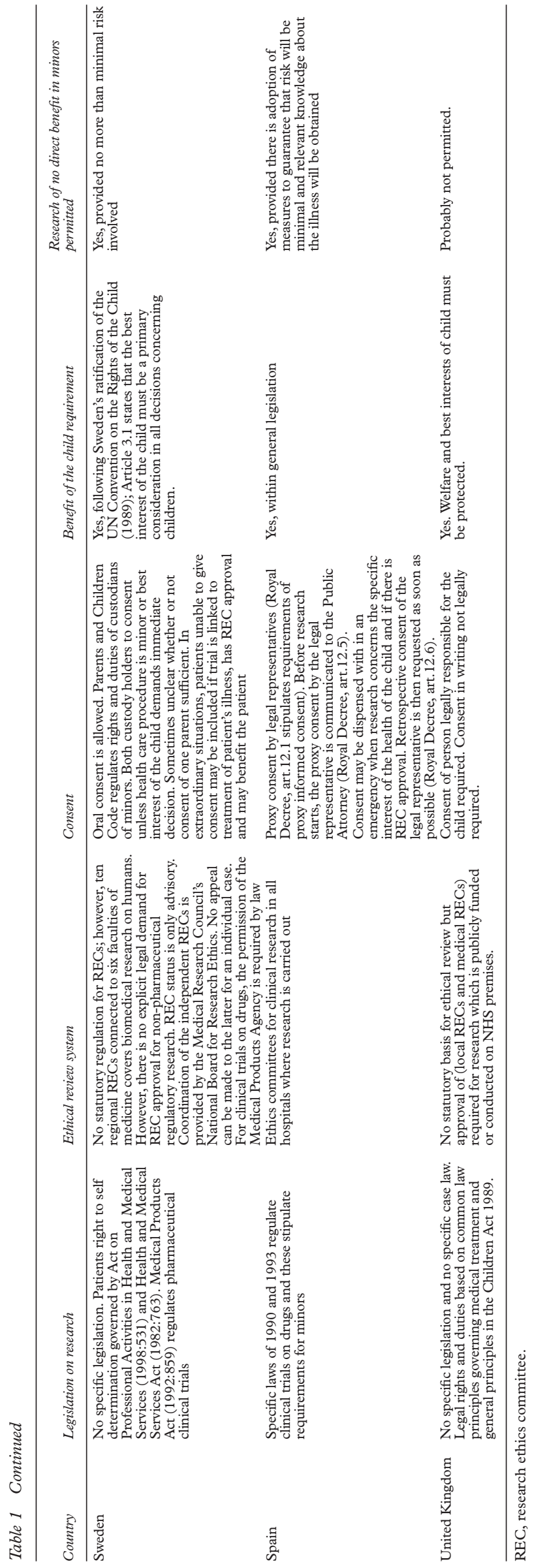

similarities in five areas governing informed consent for neonatal research across ten European countries are summarised in table 1 . A wider discussion of the differing legislation and its implications for neonatal research will be presented elsewhere. ${ }^{6}$

LEGISLATION ON RESEARCH

Six of the ten countries examined (Germany, Greece, Ireland, Norway, Spain, and Sweden) have specific legislation governing pharmaceutical trials. The laws of Denmark, Finland, and France cover other types of research as well, and the United Kingdom has no legislation specific to research but relies on general principles of law, for example, governing protection against bodily harm.

ETHICAL REVIEW SYSTEM

Research involving humans is reviewed independently by multidisciplinary research ethics committees in all countries except Greece, where this is carried out more usually by scientific committees (which are not multidisciplinary). Here, as in Norway and the United Kingdom, review is not a statutory requirement, as it is for all research in Denmark, Finland, and France. In Germany, Ireland, Spain, Sweden, and Greece, research ethics committee review is a legal requirement for clinical trials involving medicinal products; for the last country, control is by the National Drugs Organisation.

CONSENT

Proxy consent by legal guardians is the norm for neonatal research. Five countries accept this from one adult, but in Denmark, if parents share custody, consent from both adults is necessary, otherwise the research cannot be conducted. In Finland and Germany, only in exceptional circumstances when emergency treatment is required, can consent from one parent be accepted. In France and Sweden, consent from both parents is the norm.

Most countries permit enrolment into research without consent where this is not feasible, as part of emergency care. This is not allowed in Denmark, nor Ireland where legislation on consent for research is stringent.

In Ireland, a further legal stipulation requires that, after informed consent to a clinical trial, a six day period should elapse before the trial can be started. This provision can be amended, if dictated by the trial protocol, by application to and with the approval of the Irish Medicines Board.

\section{REQUIREMENT FOR BENEFIT OF THE CHILD}

All ten countries require a potential of direct benefit for a child to be entered into a trial or research study, either through general or specific research legislation. Thus proxy consent is not without some legal limitation.

Clinical trials specifically provide legal uncertainty because, owing to their necessary condition of therapeutic equipoise, it follows that at the time of recruitment it is unknown whether a treatment arm will confer benefit, no benefit, or perhaps even harm to the patient.

RESEARCH OF NO DIRECT BENEFIT PERMITTED

Linked to the requirement for direct benefit to the child is the issue of whether a country's law will permit research of no direct benefit to children (so called non-therapeutic research).

Most of the ten countries will permit research of no direct benefit in minors, with the stipulation that it must involve no more than minimal risk (an uncertain and subjective criterion).

Finland, France, Greece, Norway, and Spain follow the Convention for the Protection of Human Rights and permit a wider community and scientific viewpoint, 
allowing research of no direct benefit if the child's inclusion may result in increased knowledge about the illness to the benefit of other children (but again provided that no more than minimal risk is involved).

Research of no direct benefit in children is not permitted in Germany and Ireland.

\section{Discussion}

Increasingly, European countries collaborate in research projects. This is due to scientific ties and cooperation between various European research centres and also to the need for multinational research which is increasingly required in order to recruit sufficient patients to attain results with statistical significance.

Although European countries share a common cultural heritage and many similarities in their legal regulations of various subjects, they also have many differences in their legal systems, their laws, their codes of practice, and even practice itself. In research, this is reflected in the Declaration of Helsinki which is generally accepted in Europe, while specific types of research, such as that involving minors or emergency research, are not regulated in the same way in different countries. Even in countries where regulation seems to be similar, there may be differences in the interpretation, based in some cases on court decisions or official guidance, and in others in the cultural background of those involved in research. Doctors who conduct research are not always aware of the legal framework and it is the research ethics committees that have to examine the legality along with the ethical acceptability of the research project, and their practice differs in turn.

The European Union and the Council of Europe are working in the direction of harmonisation of the existing legislation and professional rules on research in humans. The 1997 Convention for the Protection of Human Rights and Dignity of the Human Being with regard to the application of biology and medicine has already been signed by 28 countries and ratified by six, proceeding to become their internal law, and it is expected that more countries will do so in the near future. Also the protocol on research which will be an adjunct to the Convention is almost ready and this will give guidance to researchers all over Europe.

\section{CONCLUSION}

Parents and babies must be given adequate legal and ethical protection with regard to research. At the same time, investigators need to be allowed to try to answer important research questions without excessive restriction, because the answers are likely to help improve neonatal care. ${ }^{78}$

Multinational research is increasingly required so that sufficient patients are recruited to attain the statistical power to answer relevant research questions within a meaningful time frame. The legislative application of ethical principles varies across Europe. This environment of legal variety in the matter of proxy consent for neonatal research results in differing practical implications for the conduct of pan-European research.

Euricon study group:

Management team:

Dr Su A Mason, Northern and Yorkshire Clinical Trials and Research Unit, University of Leeds, UK

Dr Chris Megone, Department of Philosophy, University of Leeds, UK

Mr Peter J Allmark, Faculty of Medicine, University of Sheffield, UK

Professor Dag Bratlid, University Hospital, Trondheim, Norway

Professor Pangiota Dalla-Vorgia, University of Athens Medical School, Greece

Dr A Bryan Gill, Leeds General Infirmary, UK

Mrs Patricia Morrogh, University College, Cork, Eire
Mrs Angela Phillips, Northern and Yorkshire Clinical Trials and Research Unit, University of Leeds, UK

PD Dr Stella Reiter-Theil, Centre for Ethics and Law in Medicine, University of Freiburg, Germany

The late Mr Craig Sykes, Northern and Yorkshire Clinical Trials and Research Unit, University of Leeds, UK

Legal representatives:

Judge C Byk, 62 Bd de Port-Royal, Paris, France

Professor C Casabona, Catedra de Derecho y Genoma Humano, Bilbao, Spain

Dr Ciaran Craven, Law School, Trinity College, Dublin, Ireland

Professor P Dalla-Vorgia, University of Athens Medical School, Greece

Dr Marit Halvorsen`, Institutt for Offentlig Rett, Oslo, Norway

Dr Hans-Georg Koch, $\star$ University of Freiburg, Germany

Ms Salla Lötiönen University of Helsinki, Finland

Professor Linda Nielsen, ${ }^{\star}$ University of Copenhagen, Denmark

Dr Aurora Plomer, University of Leeds, UK

Associate Professor E Rynning Uppsala University, Sweden

Ms E Gording Stang, Institutt for Offentlig Rett, Oslo, Norway

^Provided legal information but not in attendance at Euricon legal colloquium.

Partnership:

Dr Vineta Fellman, University of Helsinki, Finland

Dr Gorm Greisen, National University Hospital Copenhagen, Denmark

Dr Lena Hellstrom-Westas, University Hospital, Lund, Sweden

Dr Soren Holm, University of Copenhagen, Denmark

Professor Anne Langlois, Département d'Ethique Médicale, Créteil, France

Dr Giuseppe Latini Azienda, Ospedaliera A Di Summa, Brindisi, Italy

Dr Neena Modi, Hammersmith Hospital, London, UK

Professor Manuel Moya, Hospital Universitario San Juan, Spain

Professor Manuel Moya, Hospital Universitario San Ju

Dr Aurora Plomer, Faculty of Law, University of Leeds, UK

Dr Aurora Plomer, Faculty of Law, University of Le

Dr Glyn Russell, St Michael's Hospital, Bristol, UK

Dr C Anthony Ryan, Cork Regional Hospital, Eire
Dr Elie Saliba, University Hospital, Tours, France

Dr Elie Saliba, University Hospital, Tours, France Oslo, Norway

Dr A Michael Weindling, Liverpool Women's Hospital, Liverpool, UK

The late Professor Nils Svenningsen, University Hospital, Lund, Sweden

P DALLA-VORGIA

Medical School, University of Athens, Greece pdalla@cc.uoa.gr

S MASON

Northern and Yorkshire Clinical Trials and Research Unit,

University of Leeds, UK medsam@leeds.ac.uk

C MEGONE

Department of Philosophy, University of Leeds, UK

P ALLMARK

Department of Nursing, University of Sheffield, UK

D BRATLID

Department of Pediatrics, University Hospital, Trondheim, Norway

A B GILL

Department of Neonatology, Leeds, UK

P MORROGH

University College, Cork, Ireland

A PLOMER

Faculty of Law, University of Leeds, UK

S REITER-THEIL

Centre for Ethics in Medicine and Law, University of Freiburg, Germany on behalf of the Euricon Study Group

1 Mason SA. Obtaining informed consent for neonatal randomised controlled trials: an "elaborate ritual"? Archives of Disease in Childhood 1997;76:F1435.

World Medical Assembly. The Declarations of Helsinki. Ferney-Voltaire, France: The Association, 1964; amended in 1975, 1983, 1989 and 1996.

3 International Conference on Harmonisation. Guidelines for Good Clinical Practice (ICH GCP). London: The Association of the British Pharmaceutical Industry, 1997.

4 Council of Europe. Convention for the Protection of Human Rights and Dignity of the Human Being with Regard to the Application of Biology and Medicine: Convention on Human Rights and Biomedicine. European Treaty Series, No 164. Oviedo: Council of Europe, 1997.

5 McHaffie HE, Cuttini M, Brolz-Voit G, et al. Withholding/withdrawing treatment from neonates: legislation and official guidelines across Europe. 7 Med Ethics 1999;25:440-6.

6 Megone C, Mason SA, eds. Informed consent in European neonatal research. Aldershot: Ashgate Publishing Ltd, 2000 (in press).

7 Royal College of Paediatrics and Child Health. Safeguarding informed parental involvement in clinical research involving newborn babies and infants. A position statement. London: Royal College of Paediatrics and Child Health, 1999.

8 Bonati M, Choonara I, Hoppu K, Pons G, Seybert H. Closing the gap in drug therapy. Lancet 1999;353:1625. 